\title{
Population based analysis of surgical care for diverticulitis
}

\begin{abstract}
Background: Surgical treatment of diverticulitis lacks clear and consistent guidelines leading to potential variability based on treatment location. Our aim was to evaluate and explain geographic variability in resection rates for diverticulitis.

Methods: We performed a retrospective review of all-payer state hospital discharge data from 20 U.S. states merged with surgeon distribution data from the American Board of Medical Specialties. Resection rates were calculated for a total population of 137,793,346 in 687 counties. Then, we identified factors associated with high surgical resection rates by merging the county data resection rates with diverticular disease burden rates, surgeon distribution data from the American Board of Medical Specialties, U.S. Census data, and the Dartmouth Atlas of Healthcare Resource Files.
\end{abstract}

Results: Among the 362,401 total inpatient diverticulitis discharges, 326,437 (90.1\%) were uncomplicated, and 35,964 (9.9\%) were complicated. A total of 80,546 resections were performed $(22.7 \%)$. Mean total resection rate varied among states from a low of $13 \%$ in West Virginia to a high of $31 \%$ in Washington. Multivariate analysis revealed an association between surgeon practice location and increased likelihood of resection. There was no correlation between the resection rate and county diverticular disease burden $(p<0.01$ for both analyses).

Conclusion: Geographic variation in resection rates for diverticulitis may be influenced by the availability of surgeons rather than disease rates. These results suggest further standardization is warranted to identify evidence based guidelines for surgical intervention.
Volume 7 Issue $3-2017$

\author{
Douglas Johnston, Caitlin Stafford, David J \\ Schoetz, Todd Francone, Peter W Marcello, \\ Patricia L Roberts, Rocco Ricciardi \\ Department of Colon \& Rectal Surgery, Lahey Hospital \& \\ Medical Center, USA
}

Correspondence: Rocco Ricciardi, Department of Colon and Rectal Surgery, Chief, Colorectal Surgery Massachusetts General Hospital I5 Parkman St WACC 460 Boston, MA 02I I4, USA, Tel 78I-744-778I, Fax 78I-744-295,

Email rocco.ricciardi@lahey.org

Received: June 20, 2017 | Published: August 02, 2017

\section{Introduction}

The surgical treatment of diverticulitis is likely variable based on a number of factors including patient comorbidity, patient wishes, disease factors, and surgeon factors. Elective prophylactic colectomy has historically been recommended for patients with multiple bouts of diverticulitis, with or without complicated disease, but guidelines have changed considerably. ${ }^{1}$ The newest ASCRS practice parameters suggest the old model of diverticulitis as a progressive disease, in which recurrences predict poor outcomes, may be outdated. ${ }^{2,3}$ As such, elective colectomy for younger patients $(<50$ years $)$ with one attack of diverticulitis is no longer recommended. ${ }^{2}$ In addition, though nonoperative treatment with antibiotics is still recommended, the newest ASCRS guidelines acknowledge that the necessity of antibiotics has been called into question by recent research., ${ }^{2,6}$ Although new data have led to some of these shifts in recommendations, variation in disease presentation and clinical course as well as provider judgment rather than any generalized protocol are likely to lead to confusion regarding care paths., ${ }^{2,7}$ Thus, given the lack of clear consensus guidelines for surgery, the surgical care of diverticulitis is likely to be variable and nonstandard.

Mounting evidence suggests that recurrence rates among diverticulitis patients with uncomplicated disease are lower than previously reported, supporting the notion that indications for elective colectomy should be reconsidered. ${ }^{8,9}$ There is also evidence that the risks of readmission and emergency surgery are lower than previously reported for patients whose uncomplicated diverticulitis is nonoperatively managed, similarly supporting non-operative care in this population. ${ }^{10}$ New evidence suggests that there is no increased risk of conversion from laparoscopy among patients whose colectomies were delayed. ${ }^{11}$ In addition, others have advocated for less invasive management for even complicated diverticulitis in hemodynamically stable patients. ${ }^{12,13}$

Due to the subjective nature of the clinical decisions surrounding surgical intervention in diverticulitis, the aim of the present study was to examine variability in the rate of surgical colectomy for diverticulitis. In addition, we sought to determine potential drivers of high colectomy rates. In this manner, we hope to better understand those factors that drive surgeons and patients to proceed with surgical care in diverticular disease. A better understanding of these factors would further fortify guidelines and recommendations from surgical societies in the management of diverticulitis. ${ }^{14}$

\section{Materials and methods}

\section{Data source-AHRQ}

We obtained state discharge data from the Agency for Healthcare Research and Quality (AHRQ). ${ }^{14}$ The agency works to disseminate and provide resources for national healthcare data. These data included county-level place of residence information for: Arizona, Colorado, Florida, Iowa, Kentucky, Maryland, Michigan, North Carolina, Nebraska, New Jersey, New York, Oregon, Rhode Island, South Carolina, Utah, Vermont, Washington, Wisconsin, and West Virginia. California state hospital discharge data was obtained from the Office of Statewide Health Planning and Development in California. We signed a data-use agreement with AHRQ and the California Office of Statewide Health Planning and Development. Our study protocol 
was considered exempt by the Lahey Hospital \& Medical Center Institutional Review Board.

\section{Data source-U.S. Census}

We used U.S. census data from the 2000 U.S. Census to identify county level demographics for all counties sampled..$^{15}$ Specifically, we abstracted the following county level demographic variables: population total, persons per household, percent over 65 years of age, percent female, percent Black, percent owning own home, and percent who speak a language other than English.

\section{Data source-ABMS}

Surgeon practice location data were obtained from the American Board of Medical Specialties (ABMS), which works in collaboration with 24 specialty Member Boards to maintain the standards for physician certification. These data were paired by county code with initial population data obtained from census data. Pairing was unsuccessful for 205 out of a total of approximately 30,000 surgeons nationwide, and as a result those surgeons' location data was omitted.

\section{Data source-dartmouth atlas of healthcare}

Regional data for an additional five variables were obtained from Dartmouth Atlas of Healthcare. ${ }^{16}$ The Dartmouth Atlas Project uses Medicare data to document variations in how medical resources are distributed and used in the United States. These data from 2006 were organized by Hospital Service Area (HSA) and were merged using a county code crosswalk to aggregate values from multiple HSAs into county-level data that was paired with the initial population data set.

\section{Study population}

We used International Classification of Diseases (ICD-9) diagnostic codes to identify all patients discharged with a primary or secondary diagnosis of diverticulitis (ICD-9 code 562.11 or 562.13) from January 1, 2002 through December 31, 2004 in the 20 state inpatient data files. Patients with only diverticulosis and those with small bowel diverticulitis were not included. We defined complicated diverticular disease as patients with an abscess 569.5; fistula 569.81, 619.1, 569.1; or perforation 569.83. Additional variables were also grouped by ICD-9 codes, and included resections of the colon and/or rectum $(45.73,45.74,45.75,45.76,45.79,45.62$, and 48.63$)$.

\section{Data merge}

Of 1100 counties in the 20 states for which we had state discharge data, 45 counties were excluded due to sparse data. After aggregating county data into three-year, age-adjusted rates of diverticulitis incidence, we then identified those with a population of at least 20,000. This strategy resulted in a total of 687 counties with a population of $137,793,346$, of which approximately 104.1 million people were above the age of 18 . The data merge was performed at the level of patients' county of residence. The master file recorded county level information for: 1) diverticulitis cases from the State Inpatient Discharge Data by county, 2) population data from the U.S. Census, and 3) surgeon practice location data from the American Board of Medical Specialties (ABMS). Additional variables from the Dartmouth Atlas included: 1) acute care beds per 1,000 population, 2) primary care physicians per 100,000 population, 3 ) gastroenterologists per 100,000 population, 4) physician reimbursement per enrollee, and 4) medical discharges per Medicare beneficiaries.

\section{Resection rate variables}

Overall resection rates by county were calculated based on the total number of patients receiving at least one resection over the three-year period (2002-2004) per total patients with a diagnosis of diverticulitis over the same time period. Separate resection rates were then calculated for patients with complicated and patients with uncomplicated disease. That is, the rate of resection among patients with abscess, fistula or perforation was calculated, as well as the rate of resection among patients with none of those concurrent diagnoses. As such, the resection rate for patients with uncomplicated disease can be considered a rate of elective resection among diverticulitis patients.

\section{Statistical analysis}

Complicated surgery rate, and total ABMS surgeon data were divided into tertiles for analysis. For the complicated surgery rate, values between $0 \%$ and $59 \%$ were considered "low," between $59 \%$ and $74.9 \%$, "medium," and above $74.9 \%$, "high." County data for total ABMS surgeons were parsed into categorical tertiles with the following designations: counties with 0 surgeons, between 1 and 5 surgeons, and more than 5 surgeons. County data for ASCRS surgeons were parsed into two groups: counties with 0 ASCRS surgeons and counties with one or more ASCRS surgeons. Additionally, diverticulitis disease rates were divided into tertiles for comparison with resection rates. The three groups: low, medium, and high, were associated with disease rates as follows: 7.5 to 69.9 cases per 100,000 population for the low group, 70.1 to 96.2 for medium, and 96.3 to 332.2 for high.

Two linear regression models were created using SAS 9.3 (SAS Institute, Cary, NC). Resection rates (Overall and Uncomplicated) were entered as the dependent variable. Diverticular disease rates, surgeon practice location data and additional socioeconomic factors were used as covariates. We entered the following as continuous variables: population per U.S. census, diverticulitis rate, acute care beds per 1,000 population, PCPs per 100,000 population, gastroenterologists per 100,000 population, physician reimbursement per enrollee, persons per household, percent over 65, percent female, percent Black, percent owning own home, and percent speaking a language other than English. Resection rate for complicated disease, ABMS surgeons, and ASCRS surgeons were entered as categorical variables. The first model set the total diverticulitis disease resection rate as the dependent variable, while the second model used only the uncomplicated disease resection rate as the dependent variable. $\mathrm{P}$ values less than 0.05 were considered significant (Table 1).

Reproduced with the permission of the American Board of Medical Specialties (ABMS). All rights reserved.

\section{Results}

\section{All counties diverticulitis data}

Our analysis includes a total population of 137,793,346 from 687 counties in 20 states. Between 1/1/2002 through 12/31/2004, there were 362,401 total hospital discharges coded with a primary or secondary diagnosis of diverticulitis. Among these discharges, 326,437 $(90.1 \%)$ were classified as uncomplicated and 35,964 (9.9\%) were complicated. A total of 80,546 resections of the lower gastrointestinal tract, including the colon and/or rectum, were performed on these patients for a total resection rate of $22.7 \%$ among all diverticulitis admissions. The mean resection rate among complicated disease patients was $64 \%$, while the mean resection rate among patients with uncomplicated disease was $18.4 \%$.

Mean total resection rate varied among states from a low of $13 \%$ in West Virginia to a high of $31 \%$ in Washington state (Figure 1a). 
Resection rates among patients with complicated disease ranged from $53 \%$ in Arizona to $73 \%$ in Nebraska (Figure 1b), the rate for patients with uncomplicated disease ranged from $10 \%$ in West Virginia to $25 \%$ in Washington state (Figure 1c). County-level overall resection rates ranged from $2 \%$ to $50 \%$ with a mean of $23 \%$. For uncomplicated disease the resection rate was $0 \%$ to $39 \%$ with a mean of $18 \%$. For complicated disease the range was $0 \%$ to $100 \%$ with a mean of $64 \%$.
The American Board of Medical Specialties surgeon data revealed 14,830 total general surgeons in the study area, with a county mean of 22 surgeons (range 0-870), while ASCRS data showed 805 total colorectal surgeons with a county mean of 1 (range $0-62$ ) (Figure 2a $\& 2 b)$.

Table I Summary statistics for 20 states, including 687 counties

\begin{tabular}{|c|c|c|c|}
\hline Variable & Mean & Median & Standard deviation \\
\hline Population & 200573 & 64634 & 500305 \\
\hline Diverticulitis Cases per 100,000 population & 90.1 & 86.8 & 37.1 \\
\hline Overall Resection Rate (Percent & 22.7 & 23 & 7.9 \\
\hline \multicolumn{4}{|l|}{ Diverticulitis Patients Receiving Resection) } \\
\hline Percent Complicated Disease Patients & 64.1 & 65 & 18.7 \\
\hline \multicolumn{4}{|l|}{ Receiving Resection } \\
\hline Percent Uncomplicated Disease & 18.5 & 18.1 & 7.1 \\
\hline \multicolumn{4}{|l|}{ Patients Receiving Resection } \\
\hline ABMS Surgeons & 21.6 & 6 & 54.4 \\
\hline ASCRS Surgeons per County & 1.2 & 0 & 4.2 \\
\hline Acute Care Beds per & 12.7 & 7.2 & 15.6 \\
\hline \multicolumn{4}{|l|}{ I,000 population } \\
\hline Primary Care Physicians per & 403 & 225 & 585 \\
\hline \multicolumn{4}{|l|}{100,000 population } \\
\hline Gastroenterologists & 17.9 & 9 & 28.1 \\
\hline \multicolumn{4}{|l|}{ per 100,000 population } \\
\hline Physician Reimbursement per & 1854 & 1778 & 46.4 \\
\hline \multicolumn{4}{|l|}{ Enrollee 2004} \\
\hline Persons per Household & 2.6 & 2.5 & 0.2 \\
\hline Percent Over 65 & 14 & 13.6 & 3.6 \\
\hline Percent Female & 50.4 & 50.6 & 1.6 \\
\hline Percent Black & 8.7 & 3.2 & 12.6 \\
\hline Percent Owner & 71.9 & 73.4 & 8.3 \\
\hline Percent Speak Language Other than English & 9.8 & 6 & 10.5 \\
\hline
\end{tabular}

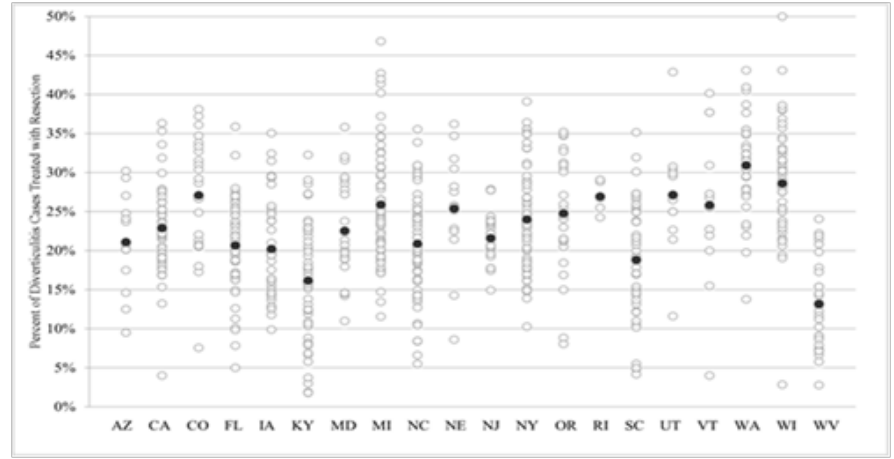

Figure Ia State variation in overall resection rate for diverticulitis patients. Dark black circle represents the state mean for all years and counties.

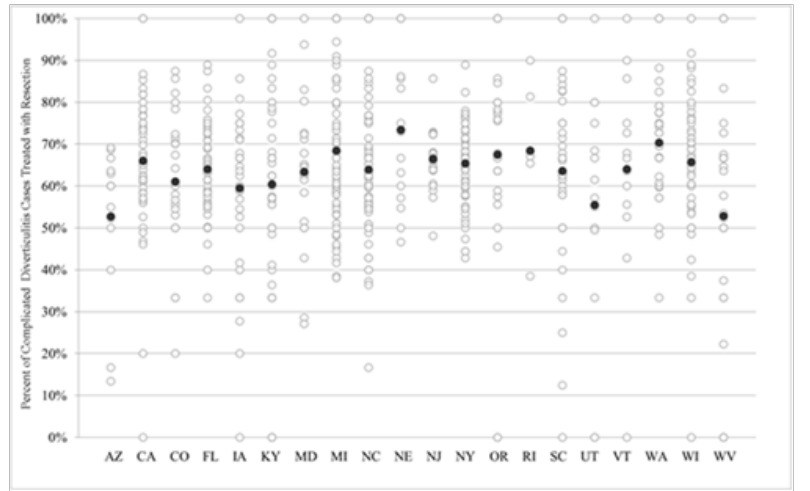

Figure Ib State variation in resection rate for diverticulitis patients with complicated disease. Dark black circle represents the state mean for all years and counties. 


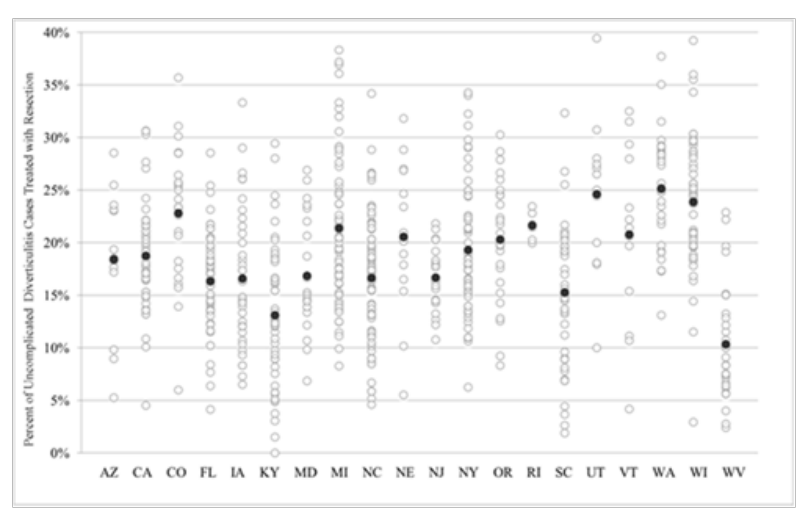

Figure Ic State variation in resection rate for diverticulitis patients with uncomplicated disease. Dark black circle represents the state mean for all years and counties.

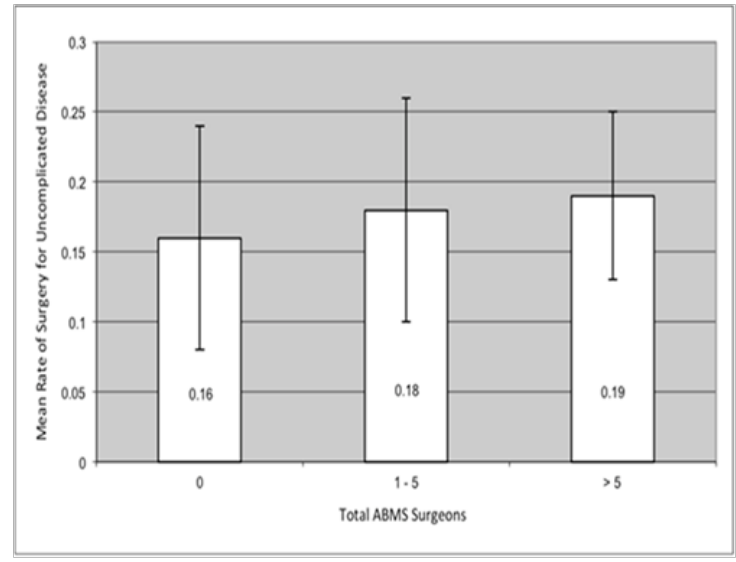

Figure $2 \mathbf{a}$ The mean resection rate among patients with uncomplicated disease increased with total number of ABMS surgeons in counties with $0, I$ to 5 , and more than 5 total surgeons. Error bars indicate \pm I standard deviation.

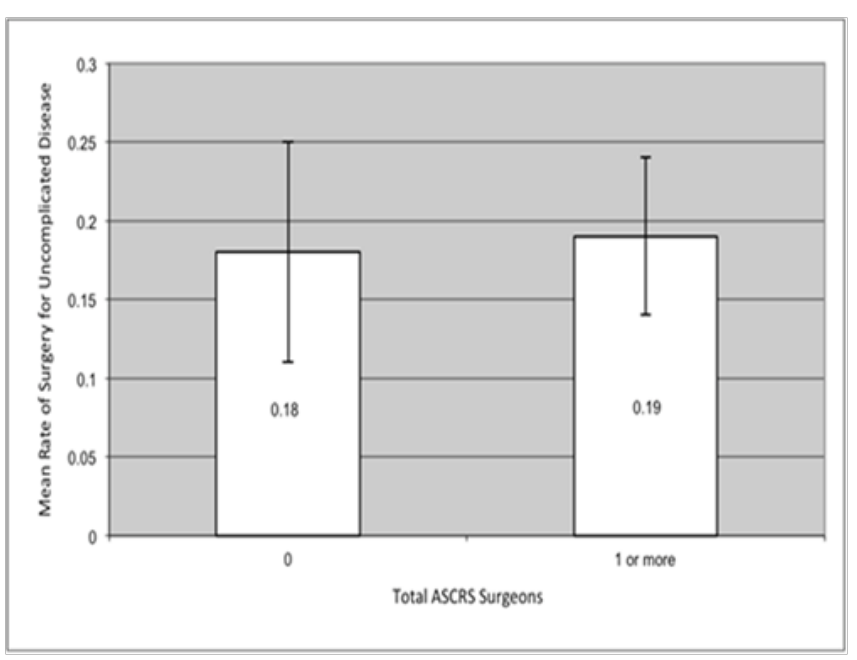

Figure $\mathbf{2 b}$ The mean resection rate among patients with uncomplicated disease increased with total number of ASCRS surgeons between counties with a total of 0 and I or more ASCRS surgeons. Error bars indicate \pm 1 standard deviation.

\section{Multivariate analysis}

Two linear regression models constructed with dependent variables of overall resection rate and uncomplicated disease resection rates were constructed (Table $2 \& 3$ ). We noted a significant association between overall resection rate and increasing ABMS surgeon tertile $(\mathrm{p}=0.02)$, as well as a significant inverse association with diverticulitis disease rate $(\mathrm{p}<0.01)$ (Table 2). Uncomplicated disease resection rate was also significantly positively correlated with increasing ABMS surgeon tertile $(\mathrm{p}=0.05)$ and inversely associated with diverticulitis disease rates $(p<0.01)$ (Table 3$)$. Thus, diverticular disease burden did not seem to drive more surgical resections.

Table 2 Linear regression analysis for overall resection rate (surgeries per diverticulitis patient)

\begin{tabular}{llll}
\hline Variable & Parameter estimate & tValue & p Value \\
\hline Population & $-9.8 \times 10^{\wedge}-13$ & 0 & 1 \\
Rate of Diverticulitis & $-6.4 \times 10^{\wedge}-4$ & -8.27 & $<0.0 I^{*}$ \\
Resection Rate Tertile for Complicated Disease & $2.6 \times 10^{\wedge}-2$ & 7.51 & $<0.0 I^{*}$ \\
Category of ABMS Surgeons & $1.1 \times 10^{\wedge}-2$ & 2.44 & $0.02^{*}$ \\
Category of ASCRS Surgeons per County & $9.3 \times 10^{\wedge}-3$ & 1.27 & 0.2 \\
Acute Care Beds per I,000 population & $-3.4 \times 10^{\wedge}-3$ & -5.5 & $<0.0 I^{*}$ \\
Primary Care Physicians per 100,000 population & $6.9 \times 10^{\wedge}-5$ & 3.51 & $<0.0 I^{*}$ \\
Gastroenterologists per 100,000 population & $3.7 \times 10^{\wedge}-4$ & 1.07 & 0.3 \\
Physician Reimbursement per Enrollee 2004 & $6.8 \times 10^{\wedge}-6$ & 0.86 & 0.4 \\
Persons per Household & $9.8 \times 10^{\wedge}-4$ & 0.04 & 1 \\
Percent Over 65 & $-6.2 \times 10^{\wedge}-4$ & -0.51 & 0.6 \\
Percent Female & $-2.8 \times 10^{\wedge}-3$ & -1.54 & 0.1 \\
Percent Black & $-8.9 \times 10^{\wedge}-4$ & -3.81 & $<0.01 *$ \\
Percent Owner & $3.1 \times 10^{\wedge}-4$ & 0.56 & 0.6 \\
Percent Speak Language Other than English & $-1.1 \times 10^{\wedge}-3$ & -2.16 & $0.03 *$ \\
\hline
\end{tabular}

*Indicates statistically significant result with $\mathrm{P}<0.05$. 
Table 3 Linear regression analysis for resection rate among patients with uncomplicated disease

\begin{tabular}{llll}
\hline Variable & Parameter estimate & tValue & p Value \\
\hline Population & $-7.5 \times 10^{\wedge}-10$ & -0.12 & 0.9 \\
Rate of Diverticulitis & $-5.5 \times 10^{\wedge}-4$ & -7.51 & $<0.0 I^{*}$ \\
Resection Rate Tertile for Complicated Disease & $1.1 \times 10^{\wedge}-2$ & 3.47 & $<0.0 I^{*}$ \\
Category of ABMS Surgeons & $-8.3 \times 10^{\wedge}-3$ & 1.93 & $0.05^{*}$ \\
Category of ASCRS Surgeons per County & $9.5 \times 10^{\wedge}-3$ & 1.38 & 0.2 \\
Acute Care Beds per I,000 population & $-2.9 \times 10^{\wedge}-3$ & -4.94 & $<0.0 I^{*}$ \\
Primary Care Physicians per I00,000 population & 2.93 & $<0.0 I^{*}$ \\
Gastroenterologists per I00,000 population & $5.4 \times 10^{\wedge}-5$ & 1.2 & 0.2 \\
Physician Reimbursement per Enrollee 2004 & $3.8 \times 10^{\wedge}-4$ & 0.16 & 0.9 \\
Persons per Household & $1.2 \times 10^{\wedge}-6$ & 0.71 & 0.5 \\
Percent Over 65 & $1.6 \times 10^{\wedge}-2$ & -0.38 & 0.7 \\
Percent Female & $-4.3 \times 10^{\wedge}-4$ & -1.95 & $0.05^{*}$ \\
Percent Black & $-3.3 \times 10^{\wedge}-3$ & -4.1 & $<0.0 I^{*}$ \\
Percent Owner & $-9.1 \times 10^{\wedge}-4$ & 0.2 & 0.8 \\
Percent Speak Language Other than English & $1.1 \times 10^{\wedge}-4$ & -2.43 & $0.02^{*}$ \\
\hline
\end{tabular}

*Indicates statistically significant result with $\mathrm{P}<0.05$.

We observed additional significant positive associations between overall resection rates and more PCPs per 100,000 population $(\mathrm{p}<0.01)$. Overall resection rate was significantly inversely associated with acute care beds per 1,000 population $(\mathrm{p}<0.01)$, increasing population percent of Black individuals $(\mathrm{p}<0.01)$, and population percent who speak a language other than English $(\mathrm{p}>0.03)$ (Table 2).

We noted similar patterns of association with uncomplicated disease resection rate. Specifically, there were significant positive associations between uncomplicated disease resection rate and PCPs per 100,000 population $(\mathrm{p}<0.01)$. Uncomplicated disease resection rate was significantly inversely associated with acute care beds per 1,000 population $(\mathrm{p}<0.01)$, population percent Black $(\mathrm{p}<0.01)$, and population percent who speak a language other than English $(\mathrm{p}>0.02)$ (Table 3). While there was no significant association between overall resection rate and population percent female, uncomplicated disease resection rates were significantly inversely related to percent female $(\mathrm{p}<0.05)$.

\section{Discussion}

The present study suggests that overall resection rates for patients with diverticulitis are directly related to the density of board certified surgeons in patients' county of residence. Thus, patients living in counties with fewer board certified surgeons were significantly less likely to undergo resection for uncomplicated diverticulitis than patients living in counties with 5 or more board certified surgeons. In addition, inverse associations were identified between overall diverticulitis resection rates and diverticulitis disease rates as well as the presence of acute care beds, percent of the population that is Black, and percent of the population that speaks a language other than English. The importance of socioeconomic factors on diverticulitis resection rates is of considerable importance and may provide some insight as to the pathophysiology of severe disease as well other decision making in this patient population.

The American Board of Colon \& Rectal Surgeons recommends individualized decision making for surgical intervention in uncomplicated diverticulitis with more defined guidelines for acute complicated disease. 2 The lack of defined and clear indications for surgery in acute diverticulitis renders the decision-making for surgery nonstandard and subject to interpretation. This variability can be used to explain practice pattern determinants, identify downstream consequences, and guide policy. In this analysis we sought to reveal factors related to the geographic variability of resection for diverticulitis with the hope of informing the clinical decisions surrounding surgical interventions for diverticulitis.

In addition to substantial variability in surgical rates for diverticulitis, we identified considerable influence of ABMS surgeon density on the rate of diverticular resections. Other studies have also demonstrated associations between surgeons and utilization of other surgical services. ${ }^{17}$ In contrast, ASCRS surgeons (whether or not there was an ASCRS surgeon in a certain county) were not significantly related to either the total diverticulitis resection rate or the complicated disease resection rate. Although colorectal surgeons have a less pronounced presence, this relationship invites speculation about the greater role of general surgeons, rather than colorectal surgeons on surgical management for diverticulitis. It is unclear what effect subspecialty guidelines and practice patterns have on non-subspecialists. Our data imply that the clinical decision-making process among surgeons treating diverticulitis may be different depending on location of practice.

Paradoxically, the diverticulitis disease burden of a county was not associated in a proportionate manner with either overall resection rate or uncomplicated resection rate. That is, counties with high rates of diverticulitis cases would be expected to have similarly high rates of diverticulitis resections. Instead, we found the opposite scenario. The lack of a direct relationship may suggest an interaction between socioeconomic factors, disease rate, and resection rates not explained with the disease variables, provider variables, and population variables that we analyzed. Ultimately, the data seem to indicate that disease rates do not drive the overall likelihood of having surgery. These findings do corroborate the ASCRS practice parameters that indicate 
surgery should be recommended on a case-by-case basis. However, our data point to socioeconomic variables playing a central role.

It is unclear why we have such a disconnect between diverticulitis disease burden and surgical resection rates. A disconnect between disease burden and associated procedure rates for various conditions was first outlined in 1973 by Wennberg. ${ }^{18}$ Reasons for this disconnect are multiple and include: differences in disease severity, surgeon indications, patient choice, and local socioeconomic factors among other reasons. The variability in practice patterns has led the US Department of Health and Human Services to suggest that as many as $25 \%$ of procedures may not be absolutely required..$^{19}$ Ongoing debate surrounding the validity and origin of these excess procedures has continued to be a great consternation. The concern that medical care is dictated more by supplier-induced demand rather than disease burden and procedure necessity is common to these arguments. ${ }^{18-20}$ Alternatively, the lack of definitive evidence about the origin of these variations leaves open the possibility that the variations observed are driven by a disparity in use of indicated care. That is, it may be that areas that appear to be exemplars of overutilization only appear that way because they are compared to areas with baseline underutilization due to access barriers. Indeed, there is evidence that disparity in Medicare spending between regions can largely be explained by a higher frequency of physician visits in areas with higher spending. ${ }^{21}$ This finding may suggest access issues, possibly rooted in socioeconomic differences, as potential causes of these variations. Additionally, the interaction between utilization of care and patient choice has not been fully explained, though there is some evidence that satisfaction is not related to the amount of care received..$^{22}$

As markers of socioeconomic influence, the percent of the population that was Black, and percent speaking a language other than English were both inversely related to resection rates. Some research suggests Black patients present with more severe diverticulitis initially, but that lack of adequate health insurance is a stronger predictor of disease severity and surgical treatment than race. Thus, it is possible the inverse relationship between Black population and resection rates has more to do with inadequate health insurance rather than race-based or may be related to genetic, patient preference, or other provider related factors. ${ }^{23}$ Contrary to the relationship with these ethnicity-related socioeconomic variables, percent of the population who own their own home was not significantly related to either resection rate. The present study confirms the role of socioeconomic status as a driver of surgery rates but it is unclear if lack of access portends a worse prognosis for those patients who do not have surgery.

The present study is meant to broadly investigate variables influencing geographic variation in resection rates for diverticulitis. As such, it is limited in its ability to deeply address relationships between individual variables and resection rates. While the county-wide data presented here allows geographic comparison on a national scale, it concurrently limits our ability to assess the status of the individual patients included. That is, our inability to ascertain the number of attacks of diverticulitis, non-surgical treatment attempts, and varying diagnostic methods are significant limitations. Additionally, though these results show preliminary evidence that geographic distribution of surgeons may influence surgery rates, the probable interrelation between surgeon distribution and socioeconomic variables makes the relative influence of these variables difficult to parse. Also, although perforation, abscess, and fistula status are good markers of complicated disease, other factors potentially influence disease severity, which leaves open the possibility that these other factors contribute to the patterns seen here. Finally, although our diverticulitis rates were ageadjusted by county, our resection rates were not, though this likely would not change the present results. Despite these limitations, the size of this study, and the diversity in geographic areas covered strengthens the validity of the results presented here.

\section{Conclusion}

Geographic variation in resection rate for both complicated and uncomplicated diverticulitis is readily apparent. Describing the cause of this variation appears to be not a simple matter of disease burden, socioeconomic factors, or even healthcare provider availability. Rather, it is likely that a combination of factors determines the resection rate in a geographic region. While individual variation in disease presentation has stymied attempts to generalize a treatment protocol for acute diverticulitis, it is unclear that the more individualized approach advocated by some would be beneficial in the event that surgeon practice location directly affects resection rates. ${ }^{24,25}$ However, this logic contradicts the reasonable prevailing notion that individual variation in disease makes individualized treatment universally preferable. Better understanding of geographical differences in clinical judgments that lead to resection for diverticulitis could further elucidate the connection between surgeon distribution and resection rate.

\section{Disclosures}

The American Board of Medical Specialties (ABMS) has given permission to Elsevier to include its data relating to the geographic distribution of surgeons in the publication entitled "Population Based Analysis of Surgical Care for Diverticulitis." Such inclusion of ABMS data shall be in the same form, as submitted to ABMS on Feb. 20, 2017 and reproduction is by permission of ABMS.

\section{Author contribution}

Conception and Design: Ricciardi, Johnston. Acquisition of Data: Ricciardi, Johnston. Analysis and Interpretation of Data: Ricciardi, Johnston, Stafford, Francone, Schoetz, Roberts, Marcello. Drafting the Article: Ricciardi, Johnston. Critical Revision: Ricciardi, Johnston, Francone, Schoetz, Roberts, Stafford, Marcello. Final Manuscript Approval: Ricciardi, Johnston, Francone, Schoetz, Roberts, Stafford, Marcello.

\section{Acknowledgements}

The authors thank Dimitri Goldstein and Jennifer Michael for their valuable assistance with this manuscript.

\section{Conflicts of interests}

None of the authors have a conflict of interests with any institution or organization.

\section{Funding}

None.

\section{References}

1. Rafferty J, Shellito P, Hyman NH, et al. Standards committee of american society of colon and rectal surgeons. Practice parameters for sigmoid diverticulitis. Dis Colon Rectum. 2006;49(7):939--944.

2. Feingold D, Steele SR, Lee S, et al. Practice parameters for the treatment of sigmoid diverticulitis. Dis Colon Rectum. 2014;57(3):284-294.

3. Chapman JR, Dozois EJ, Wolff BG, et al. Diverticulitis: a progressive disease? Do multiple recurrences predict less favorable outcomes? Ann Surg. 2006;243(6):876-830. 
4. Chabok A, Påhlman L, Hjern F, et al. Randomized clinical trial of antibiotics in acute uncomplicated diverticulitis. $\mathrm{Br} J$ Surg. 2012;99(4):532-539.

5. Shabanzadeh DM, Wille-Jørgensen P. Antibiotics for uncomplicated diverticulitis. Cochrane database Syst Rev. 2012;11:CD009092.

6. Hall JF, Roberts PL, Ricciardi R, et al. Long-term follow-up after an initial episode of diverticulitis: what are the predictors of recurrence? Dis Colon Rectum. 2011;54(3):283-288.

7. Wieghard N, Geltzeiler CB, Tsikitis VL.Trends in the surgical management of diverticulitis. Ann Gastroenterol Ann Gastroenterol. 2016;28(281):25-30.

8. Simianu VV, Flum DR. Rethinking elective colectomy for diverticulitis a strategic approach to population health. World $J$ Gastroenterol. 2014;20(44):16609-16614.

9. Salem TA, Molloy RG, O’Dwyer PJ. Prospective, five-year follow-up study of patients with symptomatic uncomplicated diverticular disease. Dis Colon Rectum. 2007;50(9):1460-1464.

10. Li D, de Mestral C, Baxter NN, et al. Risk of readmission and emergency surgery following nonoperative management of colonic diverticulitis: a population-based analysis. Ann Surg. 2014;260(3):423-430.

11. Colorectal Writing Group for the SCOAP-CERTAIN Collaborative. The impact of delaying elective resection of diverticulitis on laparoscopic conversion rate. Am J Surg. 2015;209(5):913-918.

12. Costi R, Cauchy F, Le Bian A, et al. Challenging a classic myth pneumoperitoneum associated with acute diverticulitis is not an indication for open or laparoscopic emergency surgery in hemodynamically stable patients. A 10-year experience with a nonoperative treatment. Surg Endosc. 2012;26(7):2061-2071.

13. Moore FA, Catena F, Moore EE, et al. Position paper: management of perforated sigmoid diverticulitis. World J Emerg Surg. 2013;8(1):55.
14. Healthcare Cost and Utilization Project (HCUP) State Inpatient Databases.

15. US Census Bureau. Census Regions and Divisions of the United States. 2014.

16. The Dartmouth Institute for Health Policy and Clinical Practice. The Dartmouth Atlas of Health Care. 2015

17. Birkmeyer JD, Reames BN, Mcculloch P, et al. Understanding of regional variation in the use of surgery. Lancet. 2013;382(9898):11211129 .

18. Wennberg J, Gittelsohn. Small area variations in health care delivery. Science. 2016;182(4117):1102-1108.

19. Stano M. Further issues in small area variations analysis. $J$ Health Polit Policy Law. 1991;16(3):573-588.

20. Dunn WR, Lyman S, Marx RG. Small area variation in orthopedics. $J$ Knee Surg. 2005;18(1):51-56.

21. Fisher ES, Wennberg DE, Stukel TA, et al. The implications of regional variations in Medicare spending. Part 1: the content, quality, and accessibility of care. Ann Intern Med. 2003;138(4):273-287.

22. Fisher ES, Wennberg DE, Stukel TA, et al. The implications of regional variations in Medicare spending. Part 2: health outcomes and satisfaction with care. Ann Intern Med. 2003;138(4):288-298.

23. Lidor AO, Gearhart SL, Wu AW, et al. Effect of race and insurance status on presentation, treatment, and mortality in patients undergoing surgery for diverticulitis. Arch Surg. 2008;143(12):1160-1165.

24. Stocchi L. Current indications and role of surgery in the management of sigmoid diverticulitis. World J Gastroenterol. 2010;16(7):804-817.

25. Martin ST, Stocchi L. New and emerging treatments for the prevention of recurrent diverticulitis. Clin Exp Gastroenterol. 2011;4:203-212. 